\title{
Trajectories and Determinants of Quality of Life in Dementia with Lewy Bodies and Alzheimer's Disease
}

\author{
Marleen van de Beek ${ }^{\mathrm{a}, *}$, Inger van Steenoven ${ }^{\mathrm{a}}$, Inez H.G.B. Ramakers ${ }^{\mathrm{b}}$, Pauline Aalten ${ }^{\mathrm{b}}$, \\ Huiberdina L. Koek ${ }^{\mathrm{c}}$, Marcel G.M. Olde Rikkert ${ }^{\mathrm{d}}$, Judith Manniën ${ }^{\mathrm{e}}$, Janne M. Papma ${ }^{\mathrm{f}}$, \\ Frank Jan de Jong ${ }^{\mathrm{f}}$, Afina W. Lemstra ${ }^{\mathrm{a}}$ and Wiesje M. van der Flier ${ }^{\mathrm{a}}$ \\ ${ }^{a}$ Department of Neurology, Alzheimer Center Amsterdam, Amsterdam Neuroscience, \\ Vrije Universiteit Amsterdam, Amsterdam UMC, Amsterdam, The Netherlands \\ ${ }^{\mathrm{b}}$ Department of Psychiatry and Neuropsychology, Alzheimer Center Limburg, \\ School for Mental Health and Neuroscience, Maastricht University, Maastricht, the Netherlands \\ ${ }^{\mathrm{c}}$ Department of Geriatric Medicine, University Medical Center Utrecht, Utrecht, Netherlands \\ ${ }^{\mathrm{d}}$ Department of Geriatrics \& Radboud UMC Alzheimer Center, Radboud Institute for Health Sciences, \\ Radboud University Medical Center, Nijmegen, the Netherlands \\ ${ }^{\mathrm{e}}$ Parelsnoer Institute, Utrecht, the Netherlands \\ ${ }^{\mathrm{f}}$ Department of Neurology and Alzheimer Center, Erasmus Medical Center, Rotterdam, the Netherlands
}

Accepted 6 May 2019

\begin{abstract}
.
Background: Quality of Life (QoL) is an important outcome measure in dementia, particularly in the context of interventions. Research investigating longitudinal QoL in dementia with Lewy bodies (DLB) is currently lacking.

Objective: To investigate determinants and trajectories of QoL in DLB compared to Alzheimer's disease (AD) and controls. Methods: QoL was assessed annually in 138 individuals, using the EQ5D-utility-score (0-100) and the health-related Visual Analogue Scale (VAS, 0-100). Twenty-nine DLB patients (age 69 \pm 6 ), 68 AD patients (age $70 \pm 6$ ), and 41 controls (age $70 \pm 5$ ) were selected from the Dutch Parelsnoer Institute-Neurodegenerative diseases and Amsterdam Dementia Cohort. We examined clinical work-up over time as determinants of QoL, including cognitive tests, neuropsychiatric inventory, Geriatric Depression Scale (GDS), and disability assessment of dementia (DAD).

Results: Mixed models showed lower baseline VAS-scores in DLB compared to AD and controls $(A D$ : $\beta \pm \mathrm{SE}=-7.6 \pm 2.8$, controls: $\beta \pm \mathrm{SE}=-7.9 \pm 3.0, p<0.05)$. An interaction between diagnosis and time since diagnosis indicated steeper decline on VAS-scores for AD patients compared to DLB patients ( $\beta \pm \mathrm{SE}=2.9 \pm 1.5, p<0.1)$. EQ5D-utility-scores over time did not differ between groups. Higher GDS and lower DAD-scores were independently associated with lower QoL in dementia patients (GDS: VAS $\beta \pm \mathrm{SE}=-1.8 \pm 0.3$, EQ5D-utility $\beta \pm \mathrm{SE}=-3.7 \pm 0.4 ; D A D$ : VAS $=0.1 \pm 0.0, \mathrm{EQ} 5 \mathrm{D}$-utility $\beta \pm \mathrm{SE}=0.1 \pm 0.1$, $p<0.05$ ). No associations between cognitive tests and QoL remained in the multivariate model.

Conclusion: QoL is lower in DLB, while in $\mathrm{AD}$ QoL shows steeper decline as the disease advances. Our results indicate that non-cognitive symptoms, more than cognitive symptoms, are highly relevant as they impact QoL.
\end{abstract}

Keywords: Alzheimer's disease, dementia, dementia with Lewy Bodies, quality of Life

\footnotetext{
${ }^{*}$ Correspondence to: Marleen van de Beek, MSc, Alzheimer Center Amsterdam \& Department of Neurology, Amsterdam UMC, VU University Medical Center, P.O. Box 7057, 1007 MB Amsterdam, The Netherlands. Tel.: +31204448527; E-mail: m.vandebeek2@vumc.nl
}

\section{INTRODUCTION}

Dementia with Lewy bodies (DLB) is the second most common form of neurodegenerative dementia 
after Alzheimer's disease (AD) in older people, accounting for $4-10 \%$ of all dementia cases [1-3]. DLB is clinically characterized by cognitive decline and one or more core features including fluctuating attention, visual hallucinations, parkinsonism and rapid eye movement (REM) sleep behavior disorder (RBD) [4]. DLB differs in clinical manifestation from $\mathrm{AD}$ with more frequent non-cognitive symptoms, including motor symptoms, autonomic dysfunction and neuropsychiatric symptoms like delusions, apathy, anxiety, and depression. DLB is associated with earlier nursing home admission [5], decreased time to death [6, 7], higher healthcare costs [5], and higher caregiver burden $[8,9]$ compared to AD.

Quality of Life (QoL) is an important outcome measure in dementia, especially when evaluating the efficacy of interventions. Health-related QoL in dementia is a multidimensional concept that includes perceived mental, physical, emotional, and social functioning in relation to different stages of a disease [10]. When providing treatment in dementia care, the ultimate goal is to optimize patients' health-related QoL. Knowledge on the natural trajectory of QoL and determinants thereof is essential as this provides a baseline condition for intervention studies, but also enables the identification of potential targets for interventions aiming to optimize QoL.

To date, little is known about QoL in different types of dementia and factors that determine QoL. Reviews concerning QoL in dementia suggest that loss of functional ability, limited social engagement [11] and poorer mental and physical health [12] are associated with worse QoL, while more severe cognitive impairment does not seem to predispose for worse QoL [11]. These determinants are similar to those found in nondemented older people [13]. Concerning longitudinal QoL in dementia, some studies suggest that decline in QoL over time is associated with the presence of neuropsychiatric symptoms [12, 14-17], while another study only found this effect when QoL was caregiver reported [18]. However, these studies mainly considered QoL in AD and used nursing home populations, i.e., late disease stages. For DLB, one study found that QoL was lower in DLB than in $\mathrm{AD}$, and that this was associated with the presence of neuropsychiatric symptoms [19]. However, this study was limited by a small sample size and cross-sectional design. We hypothesized that QoL would decline faster over time in DLB than in $\mathrm{AD}$, related to more prominent involvement of non-cognitive symptoms in DLB. To test this hypothesis, the current multicenter study aimed to investigate determinants and trajectories over time of QoL in DLB, compared to $\mathrm{AD}$ and controls.

\section{METHODS}

\section{Patient groups}

In this multicenter study, we included 140 patients from the Dutch Parelsnoer Institute (PSI) Neurodegenerative Diseases $(n=110)$ [20, 21], and the Amsterdam Dementia Cohort (ADC; $n=30$ ) [22]. PSI is an ongoing collaboration of eight university medical centers in the Netherlands, in which data are prospectively and uniformly collected. Patients in PSI-neurodegenerative diseases were referred to one of the eight Dutch academic memory clinics for the evaluation of cognitive problems. The ADC is based on data from the Alzheimer Center Amsterdam, which is one of the eight memory clinics that contributes to the PSI cohort and thus follows a similar protocol as PSI-neurodegenerative diseases. For this study, the ADC provided an additional sample of subjects that were not included in the PSI cohort.

All patients received standardized work-up including clinical data, cognitive assessment, MRI, blood samples and CSF samples (optional) [20]. Syndrome diagnoses were made in multidisciplinary meetings; and included subjective cognitive decline (SCD), mild cognitive impairment (MCI), or dementia. In case of dementia, an etiological diagnosis was made. For probable DLB, etiological diagnoses were made according to the 2005 international consensus criteria [23], for AD the National Institute of Neurological and Communicative Disorders and Stroke and the Alzheimer's Disease and Related Disorders Association (NINCDS-ADRDA) criteria were used [24]. All patients were invited annually for follow-up for at least two years. In both cohorts, follow-up visits consisted of neuropsychological assessment, clinical questionnaires, and clinical assessment. Total followup duration varied per patient, the median number of visits was 3 , with a minimum of 1 visit and a maximum of 6 visits. Mean follow-up time was $2.5 \pm 1.8$ years.

Patients were eligible for inclusion if QoL data were available. From the PSI cohort, we included all 19 patients with probable DLB. We matched 58 patients with $\mathrm{AD}$ and 33 controls with SCD on age range and sex. From the ADC, we additionally included all DLB patients with available QoL data $(n=10)$ [22]. We matched these for age and sex with $10 \mathrm{AD}$ patients and $10 \mathrm{SCD}$ patients. 
The PSI-neurodegenerative diseases cohort and ADC are approved by the Medical Ethics Review Committee of the Amsterdam University Medical Center. The research is performed according to the principles of the Declaration of Helsinki. All patients enrolled in the study gave their written informed consent.

\section{Instruments}

\section{Quality of Life}

QoL was assessed with the Euro Quality of Life-5 dimensions (EQ5D) [25]. The EQ5D consists of five questions and a health-related visual analogue scale (VAS). In these questions, the respondent is asked to rate their current health state on five dimensions (mobility, hygiene, usual activities, pain/discomfort, and anxiety/depression) into one of three levels (no problems (1), some problems (2) or a lot of problems (3)). The answers are transformed into a utility value, describing the respondent's health, where utility $=0$ equals death and utility $=1$ equals perfect health. For example, the answer pattern 11111 would result in perfect health state (utility $=1$ ), whereas 33333 would result in the worst possible health state (utility $<0$, worse than death). The weights of the different answer patterns are based on the valuation of these health states by the Dutch population, making these scores useful in cost-utility analyses, healtheconomics and medication trials.

In contrast, the VAS reflects the self-perceived health-related QoL. Using the VAS, the respondents rate their own health status on a $20-\mathrm{cm}$ health thermometer, which is recoded as a score between 0 (worst imaginable health state) to $100 \%$ (best imaginably health state) [25].

\section{Non-cognitive symptoms}

DLB core criteria were assessed for DLB patients only during the clinical assessment at baseline. Core criteria of DLB, i.e., visual hallucinations, parkinsonism, fluctuations, and RBD were rated as being present or absent. Neuropsychiatric symptoms were assessed with the 12 items Neuropsychiatric Inventory, with information provided by the caregiver [26]. We recoded the neuropsychiatric symptoms as being present $(\geq 1)$ or absent. The Disability Assessment for Dementia (DAD) is administered through an informant-based interview for measuring the competence in instrumental activities in daily living ((I)ADL), with scores ranging from 0 to $100 \%$, higher scores indicating higher competence [27]. Depressive symptoms were assessed with the self-reported Geriatric Depression Scale (GDS), with scores ranging from 0 to 15 , higher scores indicating more depressive symptoms [28].

\section{Cognition}

We used the Mini-Mental State examination (MMSE) to assess global cognition [29]. Memory functioning was assessed with the Dutch version of the Auditory Verbal Learning test (AVLT). We used the summed score of direct recall after 5 presentations. The delayed recall was assessed after 20 minutes. Speed and attention were assessed with the Trail Making Test-A (TMT-A), and Trail Making Test-B (TMT-B) was used as a measure for executive functioning.

\section{Statistical analysis}

Analyses were performed in SPSS (IBM ${ }^{\circledR}$, V22, Chicago, IL) and R (version 3.2.5, R Development Core Team, 2010). After data check, we identified and removed two outliers from the control group ( $>3$ SD on baseline VAS). Hence, all analyses were conducted on 29 DLB patients, 68 AD patients, and 41 controls. EQ5D-utility scores were multiplied by 100 , so that EQ5D-utility ranges between 0 and 100. Multiple imputation was used to impute missing values of individual neuropsychological test-scores. Fifteen imputed datasets were created to ensure stability of the results. Analyses were done on the pooled datasets. For TMT-A and TMT-B, inverted data were used such that lower scores represent worse performance. We log-transformed TMT-data to meet the assumption of normally distributed data. ANOVA, Kruskal-Wallis, and $\chi^{2}$ tests were used to test for differences in demographics between DLB, AD, and controls at baseline. Linear mixed models (LMM) were used to compare QoL trajectories (EQ5D-utility and VAS as dependent variables in separate models) between groups. The models included time since diagnosis (years), diagnosis (categorical variable, DLB as reference), and interaction of time and diagnosis as independent variables. The model included a random intercept and a random slope. Main effect of diagnosis represents the association between diagnosis and QoL at baseline. The interaction term between time and diagnosis represents the association between diagnosis and annual change in QoL. The analyses were conducted with adjustment for age and sex. To assess possible determinants of QoL in dementia (DLB and AD; controls were not included in 
this analysis), we used LMM with baseline demographic variables, diagnosis, baseline and follow-up data from the NPI subscales (0-1), GDS (0-15), DAD (0-100), and cognitive test scores (MMSE, AVLT, TMT-A, and TMT-B) as independent variables and all available VAS and EQ5D-utility scores as dependent variables. Of note, we used all available data, baseline and follow-up, taking into account correlations within persons. We first constructed univariate models for each of the putative determinants and for both outcome measures. Subsequently, we constructed a multivariate model, based on significant determinants of the univariate models only (manual backwards selection, with diagnosis added as covariate). For main effects, significance level was set at $p<0.05$, for interaction effects at $p<0.10$.

\section{RESULTS}

Demographic and clinical data are presented in Table 1. MMSE and AVLT scores at baseline were lower for the DLB and AD groups compared to controls $(p<0.001)$. TMT-A and TMT-B scores were lower in DLB compared to AD and controls (AD: $p<0.05$, controls: $p<0.001)$. AD patients scored lower on TMT-A and TMT-B scores compared to controls as well $(p<0.001)$. Furthermore, patients with DLB had lower DAD scores than controls $(p<0.001)$. In line with diagnostic criteria, caregivers more frequently reported hallucinations and apathy in DLB patients compared to controls $(p<0.05$, Fig. 1). At baseline, $14 \%$ of DLB patients and $27 \%$ of $\mathrm{AD}$ patients were using cholinesterase inhibitors.

Linear mixed models showed that baseline QoL measured with the VAS was lower in DLB compared to $\mathrm{AD}$ and controls $(A D: \beta \pm \mathrm{SE}=-7.6 \pm 2.8$, controls: $\beta \pm \mathrm{SE}=-7.9 \pm 3.0$, both $p<0.05$ ) (Table 2 and Fig. 2A). In addition, we found an interaction between diagnosis and time, indicating that patients with AD had a steeper decline over time than DLB $(\beta \pm \mathrm{SE}=2.9 \pm 1.5, p<0.10)$. QoL in DLB patients remained stable (Fig. 2A).

Table 1

Baseline characteristics

\begin{tabular}{|c|c|c|c|c|c|c|}
\hline & $\mathrm{n}$ & DLB & $\mathrm{n}$ & $\mathrm{AD}$ & $\mathrm{n}$ & Controls \\
\hline Sex, n female $(\%)^{1}$ & 29 & $2(6.9 \%)$ & 68 & $2(2.9 \%)$ & 41 & $2(4.9 \%)$ \\
\hline $\mathrm{Age}^{1}$, mean $\pm \mathrm{SD}$ & 29 & $69.0 \pm 6.3$ & 68 & $70.4 \pm 6.1$ & 41 & $69.8 \pm 5.3$ \\
\hline Years of education, mean $\pm S D$ & 29 & $11 \pm 3$ & 68 & $11 \pm 4$ & 41 & $11 \pm 3$ \\
\hline Follow-up time, mean \pm SD & 29 & $2.1 \pm 1.9$ & 68 & $2.5 \pm 1.9$ & 41 & $2.9 \pm 1.8$ \\
\hline On CHEI, n (\%) & 29 & $4(13.8 \%)^{\mathrm{a}}$ & 68 & $18(26.5 \%)^{\mathrm{a}}$ & 41 & 0 \\
\hline DAD, median [range] & 27 & $79[31-100]^{\mathrm{a}}$ & 68 & $81[31-100]^{\mathrm{a}}$ & 41 & $95[33-100)$ \\
\hline GDS, median [range] & 28 & $3[1-11]$ & 68 & $2[0-11]$ & 41 & $2[0-10]$ \\
\hline NPI, median [range] & 29 & $16[0-69]$ & 68 & $11[0-75]$ & 41 & $6[0-61]$ \\
\hline \multicolumn{7}{|l|}{ Cognitive tests, median [IQR] } \\
\hline MMSE & 28 & $25[22-27]^{\mathrm{a}}$ & 68 & $25[22-27]^{\mathrm{a}}$ & 41 & $29[25-30]$ \\
\hline AVLT - 5 trials summed ${ }^{\#}$ & 28 & $23[17-30]^{\mathrm{a}}$ & 68 & $22[15-28]^{\mathrm{a}}$ & 41 & $36[30-44]$ \\
\hline AVLT - delayed recall ${ }^{\#}$ & 28 & $4[1-6]^{\mathrm{a}}$ & 68 & $2[0-4]^{\mathrm{a}}$ & 41 & $6[5-8]$ \\
\hline Trail Making Test A - time & 28 & $91[68-119]^{\mathrm{a}, \mathrm{b}}$ & 68 & $61[46-80]^{\mathrm{a}}$ & 41 & $39[35-51]$ \\
\hline Trail Making Test B - time & 28 & $324[230-480]^{a, b}$ & 68 & $188[139-280]^{\mathrm{a}}$ & 41 & $88[76-111]$ \\
\hline \multicolumn{7}{|l|}{ DLB symptoms, n(\%) } \\
\hline Fluctuations & 24 & $18(75 \%)$ & & & & \\
\hline Visual hallucinations & 24 & $15(63 \%)$ & & & & \\
\hline Parkinsonism & 24 & $16(67 \%)$ & & & & \\
\hline RBD & 17 & $10(59 \%)$ & & & & \\
\hline \multicolumn{7}{|l|}{ Quality of Life, median [IQR] } \\
\hline VAS & 27 & $70[61-80]^{\mathrm{b}}$ & 68 & $80[70-90]$ & 41 & 80 [70-90] \\
\hline EQ5D-utility & 27 & $84[72-90]$ & 68 & $90[77-100]$ & 41 & $90[80-100]$ \\
\hline Mobility & & $9(33 \%)$ & & $18(27 \%)$ & & $17(22 \%)$ \\
\hline Self-care & & $3(11 \%)$ & & $5(7 \%)$ & & 0 \\
\hline Usual activities & & $9(33 \%)$ & & $17(25 \%)$ & & $6(15 \%)$ \\
\hline Pain/discomfort & & $12(44 \%)$ & & $23(34 \%)$ & & $17(42 \%)$ \\
\hline Anxiety/depression & & $8(30 \%)$ & & $18(27 \%)$ & & $6(15 \%)$ \\
\hline
\end{tabular}

DLB, dementia with Lewy bodies; AD, Alzheimer's disease; MMSE, Mini-Mental State Examination; CHEI, cholinesterase inhibitor; DAD, Disability Assessment for Dementia; GDS, Geriatric Depression Scale; NPI, Neuropsychiatric Inventory; AVLT, Auditory Verbal Learning Test, RBD, REM sleep behavior disorder. ${ }^{\mathrm{a}} p \leq 0.05$ compared to controls, ${ }^{\mathrm{b}} p \leq 0.05$ compared to AD. ${ }^{1}$ Matched variables. ${ }^{\#}$ Imputed data. Percentage of missing values before imputation: ALVT-direct recall: $1.5 \%$, ALVT-delayed recall: $1.5 \%$, TMT-A: $1.5 \%$, TMT-B: $15.3 \%$. 
For QoL measured with EQ5D-utility, we found that DLB had lower QoL at baseline compared to controls $(\beta \pm \mathrm{SE}=-9.2 \pm 4.1, p<0.05)$. There was no significant difference in course of EQ5D-utility scores over time between groups (Table 2 and Fig. 2B). Analysis of the five separate questions of the EQ5D revealed no significant differences between groups on either the mobility, self-care, usual activities, pain/discomfort or depression/anxiety question (Table 1).

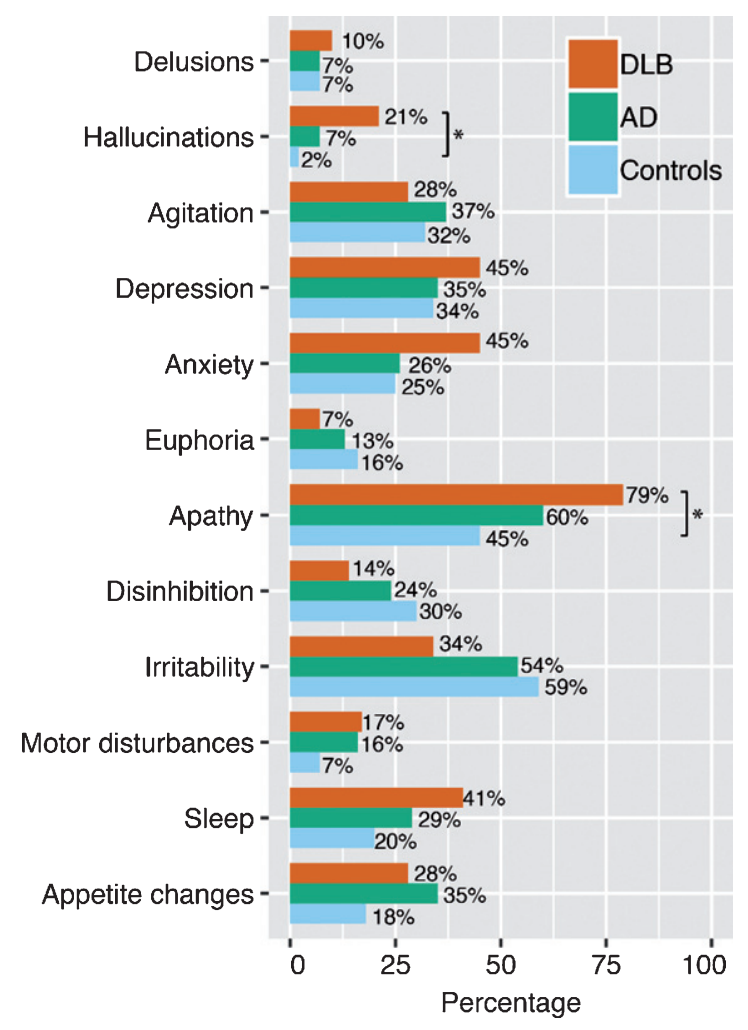

Fig. 1. The prevalence of Neuropsychiatric Inventory (NPI) symptoms in dementia with Lewy bodies (DLB, $n=29$ ), Alzheimer's disease (AD, $n=68)$, and controls $(n=41)$ at baseline. ${ }^{*} p<0.05$, $\chi^{2}$ test.
Subsequently, to assess possible determinants for QoL in dementia, we used linear mixed models. Table 3 shows the associations of different demographics at baseline, neuropsychiatric symptoms, and cognitive tests scores with QoL in terms of VAS and EQ5D. For VAS scores we found an association with diagnosis, as DLB had lower VAS scores compared to $\mathrm{AD}(\beta \pm \mathrm{SE}=5.1 \pm 2.4$, $p<0.05)$. Furthermore, higher scores on the GDS and lower scores on the DAD were associated with lower VAS score $(G D S \beta \pm \mathrm{SE}=-1.9 \pm 0.3, p<0.001$; $D A D \beta \pm \mathrm{SE}=0.1 \pm 0.0, p<0.05)$. In addition, lower TMT-A scores were associated with lower VAS scores $(\beta \pm \mathrm{SE}=3.4 \pm 1.6, p<0.05)$. Subsequently, we constructed a multivariate model using backwards selection, controlling for diagnosis. GDS and DAD scores remained as independent determinants $(G D S \beta \pm \mathrm{SE}=-1.8 \pm 0.3, p<0.001 ; D A D$ $\beta \pm \mathrm{SE}=0.1 \pm 0.0, p<0.05)$.

Lower scores for EQ5D-utility were related with higher scores on the GDS and lower scores on the DAD (GDS $\beta \pm \mathrm{SE}=-3.7 \pm 0.4$, $p<0.001 ; D A D \beta \pm \mathrm{SE}=0.2 \pm 0.1, p<0.05$ ). Furthermore, presence of depression and anxiety on NPI were associated with lower EQ5D-utility (depression $\beta \pm \mathrm{SE}=-5.6 \pm 2.5, p<0.05 ;$ anxiety $\beta \pm \mathrm{SE}=$ $5.4 \pm 2.6, p<0.05)$. In a multivariate model with backwards selection, both GDS and DAD remained independent determinants of EQ5D-utility (GDS $\beta \pm \mathrm{SE}=-3.7 \pm .4, p<0.001 ; D A D \beta \pm \mathrm{SE}=0.1 \pm 0.1$, $p<0.05)$. When stratifying the data for diagnosis, comparable results were found in AD and DLB separately (data not shown).

\section{DISCUSSION}

The main finding of this multicenter study is that QoL at the time of diagnosis was lower in DLB patients than in $\mathrm{AD}$ patients, while subsequently $\mathrm{QoL}$ in $\mathrm{AD}$ patients declined more steeply over time and

Table 2

Dementia types and trajectories in $\mathrm{QoL}$

\begin{tabular}{lccccc}
\hline & \multicolumn{2}{c}{ VAS } & & \multicolumn{2}{c}{ EQ5D-utility } \\
\cline { 2 - 3 } \cline { 5 - 6 } & Baseline effect & Change over time & & Baseline effect & Change over time \\
\hline DLB versus AD & $\mathbf{- 7 . 5 5}(\mathbf{2 . 7 9})^{*}$ & $\mathbf{2 . 8 5}(\mathbf{1 . 5 4})^{+}$ & & $-5.40(3.80)$ & $1.26(2.11)$ \\
DLB versus controls & $\mathbf{- 7 . 9 4 ( 3 . 0 2 ) ^ { * }}$ & $1.77(1.61)$ & & $\mathbf{- 9 . 1 6 ( \mathbf { 4 . 1 1 } ) *}$ & $2.95(2.23)$ \\
\hline
\end{tabular}

Data represent $\beta$ (SE). Linear mixed models were used include term for time and diagnosis, the interaction term for time * diagnosis was used to estimate change of over time between diagnosis for VAS and EQ5D-utility scores, corrected for age and sex. Negative betas indicate lower scores at baseline for DLB compared to AD/controls or steeper decline over time for DLB compared to AD/controls. ${ }^{*} p \leq 0.05,{ }^{+} p \leq 0.1$. DLB, dementia with Lewy bodies; AD: Alzheimer's disease. 

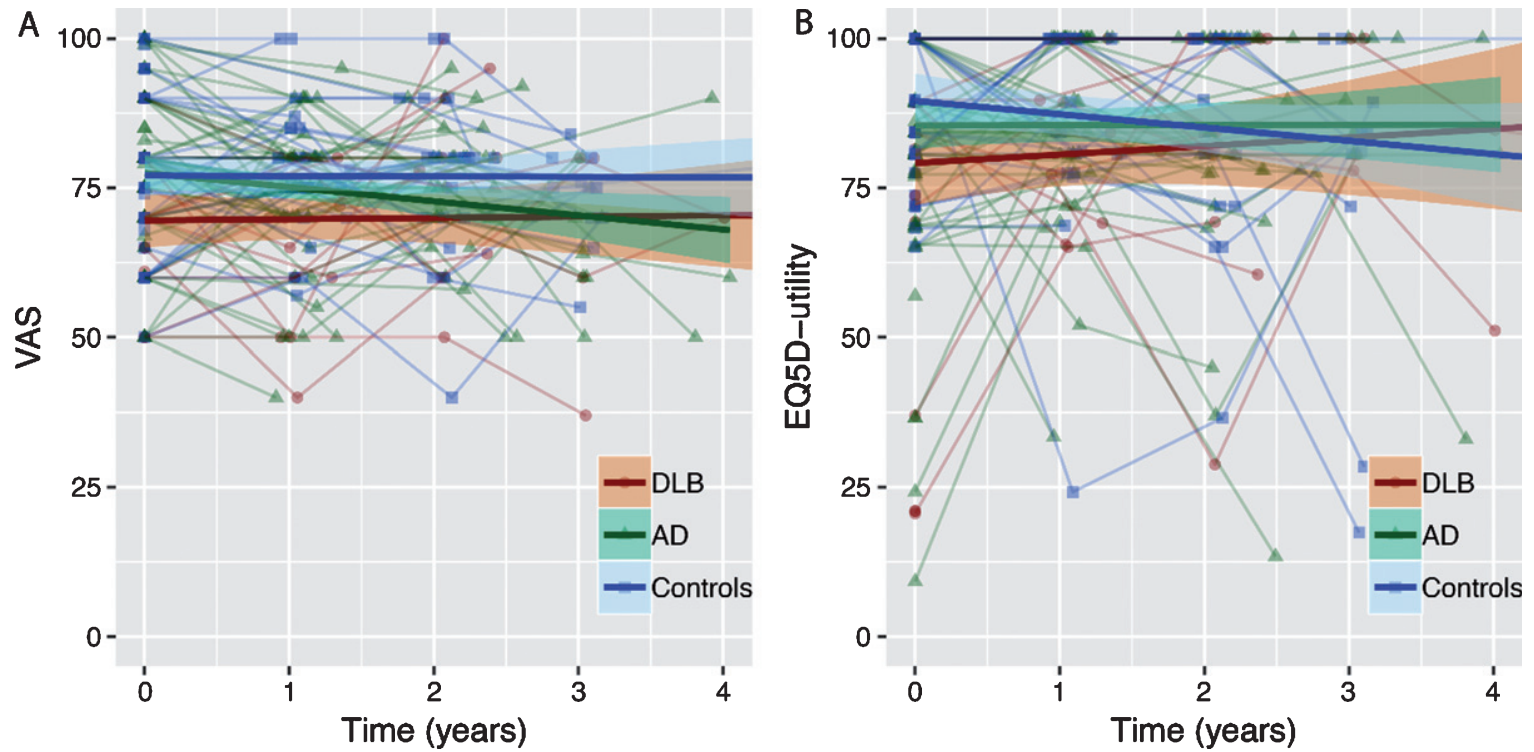

Fig. 2. Estimated trajectories of QoL over time for patients with DLB, AD, and controls. Data represent individual trajectories of raw QoLscores over time. Regression lines represent estimated group trajectories over time in years with 95\% confidence intervals. A) Trajectories of Visual Analogue Scale (VAS) over time. B) Trajectories of EQ5D-utility scores over time.

Table 3

Mixed models associations with VAS and EQ5D-utility

\begin{tabular}{|c|c|c|c|c|}
\hline & \multicolumn{2}{|c|}{ VAS } & \multicolumn{2}{|c|}{ EQ5D-utility } \\
\hline & Model 1 & Model 2 & Model 1 & Model 2 \\
\hline Diagnosis, $A D$ versus DLB & $5.06(2.37)^{*}$ & & $5.86(3.71)$ & \\
\hline Sex, female versus male & $-1.46(5.95)$ & & $-3.11(8.80)$ & \\
\hline Age, years & $0.24(0.17)$ & & $-0.07(0.26)$ & \\
\hline MMSE & $0.07(0.19)$ & & $0.06(0.27)$ & \\
\hline GDS & $-1.85(0.30)^{*}$ & $-1.79(0.32)^{*}$ & $-3.71(0.42)^{*}$ & $-3.67(0.42)^{*}$ \\
\hline DAD & $0.12(0.04)^{*}$ & $0.10(0.04)^{*}$ & $0.15(0.06)^{*}$ & $0.14(0.06)^{*}$ \\
\hline AVLT - direct recall ${ }^{\#}$ & $-0.03(0.08)$ & & $0.14(1.25)$ & \\
\hline AVLT - delayed recall ${ }^{\#}$ & $-0.51(0.36)$ & & $-0.01(0.55)$ & \\
\hline Trail Making Test $-\mathrm{A}^{\#}$ & $3.38(1.62)^{*}$ & & $1.74(2.29)$ & \\
\hline Trail Making Test $-\mathrm{B}^{\#}$ & $0.34(1.73)$ & & $2.25(2.57)$ & \\
\hline NPI subscales & & & & \\
\hline Delusions & $-1.77(2.76)$ & & $-0.66(3.98)$ & \\
\hline Hallucinations & $-2.98(2.48)$ & & $-0.16(3.57)$ & \\
\hline Agitation & $1.99(1.83)$ & & $1.29(2.61)$ & \\
\hline Depression & $-0.34(1.78)$ & & $-5.63(2.50)^{*}$ & \\
\hline Anxiety & $-2.20(1.84)$ & & $-5.37(2.60)^{*}$ & \\
\hline Euphoria & $2.34(2.60)$ & & $0.40(3.68)$ & \\
\hline Apathy & $-1.78(1.74)$ & & $-0.42(2.48)$ & \\
\hline Disinhibition & $3.50(2.13)$ & & $-0.21(3.07)$ & \\
\hline Irritability & $-0.43(1.76)$ & & $-3.34(2.51)$ & \\
\hline Aberrant motor behavior & $0.97(2.09)$ & & $-2.43(2.98)$ & \\
\hline Sleep disturbances & $-0.87(1.86)$ & & $-0.02(2.65)$ & \\
\hline Eating disturbances & $1.09(1.88)$ & & $-1.04(2.66)$ & \\
\hline
\end{tabular}

Data represent $\beta$ (SE). Linear mixed models include putative determinants as independent variables and VAS and EQ5D-utility as outcome variable. Model 1: univariate associations with VAS and EQ5D-utility. Model 2: associations with VAS and EQ5D after backwards selection. MMSE, Mini-Mental State Examination; DAD, Disability Assessment for Dementia; GDS, Geriatric Depression Scale; NPI, Neuropsychiatric Inventory; AVLT, Auditory Verbal Learning Test. ${ }^{*} p \leq 0.05$. "Missing values are imputed with multiple imputation, analyses are done on fifteen pooled datasets, percentage of missing values before imputation: ALVT-direct recall: $4.5 \%$, ALVTdelayed recall: $4.8 \%$, TMT-A: $3.1 \%$, TMT-B: $25 \%$. 
DLB patients remained relatively stable. In addition, we found that independent of type of dementia, higher reports of depressive symptoms and higher dependency in (I)ADL were associated with lower QoL, while severity of cognitive impairment was not associated with lower QoL.

At baseline, QoL in DLB patients was lower than in AD patients. Contrary to our hypothesis, however, QoL in DLB remained relatively stable on followup, while we observed marked decline in QoL as measured by VAS in AD. Previous studies on longitudinal QoL in dementia have been inconclusive. While some studies report no change in QoL after one-year follow-up [30, 31], others found only a decrease in relation with neuropsychiatric symptoms [18]. Patients with DLB present with a wide variety of non-cognitive symptoms, such as motor impairment, neuropsychiatric symptoms, and autonomic dysfunction [4]. We suspect that our findings could be explained by the fact that these symptoms are present early in DLB, therefore explaining the lower QoL at baseline. It is conceivable that non-cognitive symptoms play an increasingly large role in $\mathrm{AD}$ in the course of the disease, resulting in a decline in QoL as $\mathrm{AD}$ advances [18, 32].

In line with this hypothesis and previous research, we found an association between non-cognitive symptoms and QoL, as functional impairment and depressive symptoms were related to lower QoL. Also, we found that severity of cognitive impairment was not independently related to QoL. To put this in a broader perspective, non-cognitive symptoms like depressive symptoms and loss of functional ability are not only associated with lower QoL, other studies have linked these symptoms to higher caregiver burden [33, 34] and earlier nursing home admission [35-38] as well. Taken together, this illustrates that non-cognitive symptoms, more than cognitive symptoms are highly relevant as they have impact on the experienced QoL and disease burden of patients with dementia.

Differences in trajectories of QoL between diagnostic groups were only found when QoL was measured with the VAS score. The EQ5D-utility scores show a similar trend as the VAS, yet these differences did not reach significance. A potential explanation for this finding is the distribution of EQ5D-utility scores. When all five questions of the EQ5D are answered negatively, the score automatically reaches maximum. Therefore, the utility-score shows a ceiling effect, making the statistical analyses less powerful and easy to interpret. In contrast, the
VAS score is normally distributed and is based on a more subjective rating of QoL.

Strengths of the current study include the multicenter approach and the longitudinal design. All patients were diagnosed following a standardized diagnostic protocol which was harmonized across centers. Using data from multiple centers, contributes to the generalizability of our study. Furthermore, our identified determinants of QoL were assessed over the course of the disease, so it takes into consideration the QoL of patients with dementia at several points in time. We had a mean follow-up duration of around 2.5 years, which is considerably longer than many other recent studies investigating longitudinal QoL $[14,30]$. By using a longitudinal design, we can draw conclusions about naturalistic disease course. Furthermore, our study has more power compared to a cross-sectional design, as we consider multiple data points per participant. Among the potential limitations is that not all patients that were included at baseline had sufficient follow-up data available. Patients that were lost to follow-up had lower MMSE scores at time of diagnosis and it is likely that they were in a more advanced disease stage at the time of diagnosis. The selection toward cognitively healthier patients at follow-up may give a more favorable image of the course of QoL over time. Second, it could be argued that there is considerable overlap between the concepts of depressive symptoms and QoL. Patients that score high on the GDS are likely to have a higher score on the EQ5D depression/anxiety question as well. Therefore, presence of depressive symptoms lowers the EQ5D-utility score. However, we found the same relation with depressive symptoms for the VAS score, which is not directly targeted at measuring depression. Since comparable results are found in both measures, we believe that depressive symptoms are a robust determinant of QoL, but a certain degree of circularity is inevitable. Third, our research focused on QoL in $\mathrm{DLB}$ and $\mathrm{AD}$, but did not include other types of dementia like vascular dementia or frontotemporal dementia, nor did we take the possibility of mixed pathology into account. However, we found that non-cognitive symptoms are the main determinant of QoL, irrespective of type of dementia. We suspect that in cases with mixed pathology and other types of dementia, the same non-cognitive symptoms will influence QoL. In line with this notion, previous studies on QoL in for example vascular dementia found the same determinants of $\mathrm{QoL}$ $[39,40]$. Finally, our control group consisted of 
individuals with SCD. These patients experience memory decline, but perform within normal range on cognitive tests. Previous research has found that in individuals with SCD, higher reports of cognitive complaints are related with worse QoL in SCD [41]. It is possible that the results would have been different if we used a control group without cognitive complaints, and that our current results reflect an underestimation of the effect.

This study has important clinical implications. First, our results underline the importance of preventing and treating depressive symptoms in dementia. Clinicians need to be aware of the relevance of these symptoms and adequately address them over the course of the disease in order to maintain QoL. Furthermore, our results might imply that interventions aiming to maintain independency in daily living may help to optimize QoL. This could be via psychosocial interventions that target independency, as well as pharmacological interventions. For example, one study investigating the effect of memantine in DLB found that improved physical health generally improved the perceived QoL [42]. Thus, in the search of disease modifying treatment for dementia, the focus should not merely be on preserving cognition, but also preserving autonomy. As the ultimate goal with any intervention is to maintain or improve QoL, future interventions should also target noncognitive symptoms like depression and dependency in (I)ADL.

In conclusion, we found different trajectories of QoL in DLB compared to AD, as patients with DLB initially present with lower QoL, while AD patients show steeper decline in QoL over time. Future interventions aimed at optimizing QoL in dementia should focus on decreasing depressive symptoms and maintaining independence, as these are major determinants of QoL in dementia.

\section{ACKNOWLEDGMENTS}

This research was supported by the Parelsnoer Initiative, Parel Neurodegenerative Diseases, which is part of and funded by the Netherlands Federation of University Medical Centers and has received initial funding from the Dutch Government. Research of Alzheimer Center Amsterdam is part of the neurodegeneration research program of Amsterdam Neuroscience. The Alzheimer Center Amsterdam is supported by Alzheimer Nederland and Stichting VUmc funds. A.L. has received funding from stichting Dioraphte en from ZonMW Memorabel (project \#733050509).

Authors' disclosures available online (https:// www.j-alz.com/manuscript-disclosures/19-0041r2).

\section{REFERENCES}

[1] Kane JP, Surendranathan A, Bentley A, Barker SA, Taylor J-P, Thomas AJ, Allan LM, McNally RJ, James PW, McKeith IG (2018) Clinical prevalence of Lewy body dementia. Alzheimers Res Ther 10, 19.

[2] Hogan DB, Fiest KM, Roberts JI, Maxwell CJ, Dykeman J, Pringsheim T, Steeves T, Smith EE, Pearson D, Jetté N (2016) The prevalence and incidence of dementia with Lewy bodies: A systematic review. Can J Neurol Sci 43, S83-S95.

[3] Zaccai J, McCracken C, Brayne C (2005) A systematic review of prevalence and incidence studies of dementia with Lewy bodies. Age Ageing 34, 561-566.

[4] McKeith IG, Boeve BF, Dickson DW, Halliday G, Taylor JP, Weintraub D, Aarsland D, Galvin J, Attems J, Ballard CG (2017) Diagnosis and management of dementia with Lewy bodies Fourth consensus report of the DLB Consortium. Neurology 89, 88-100.

[5] Mueller C, Ballard C, Corbett A, Aarsland D (2017) The prognosis of dementia with Lewy bodies. Lancet Neurol 16, 390-398.

[6] Williams MM, Xiong C, Morris JC, Galvin JE (2006) Survival and mortality differences between dementia with Lewy bodies vs Alzheimer disease. Neurology 67, 1935-1941.

[7] Mueller C, Soysal P, Rongve A, Isik AT, Thompson T, Maggi S, Smith L, Basso C, Stewart R, Ballard C (2019) Survival time and differences between dementia with Lewy bodies and Alzheimer's disease following diagnosis: A meta-analysis of longitudinal studies. Ageing Res Rev $\mathbf{5 0}$, $72-80$.

[8] Svendsboe E, Terum T, Testad I, Aarsland D, Ulstein I, Corbett A, Rongve A (2016) Caregiver burden in family carers of people with dementia with Lewy bodies and Alzheimer's disease. Int J Geriatr Psychiatry 31, 1075-1083.

[9] Leggett AN, Zarit S, Taylor A, Galvin JE (2010) Stress and burden among caregivers of patients with Lewy body dementia. Gerontologist 51, 76-85.

[10] Ettema TP, Dröes R-M, De Lange J, Ooms ME, Mellenbergh GJ, Ribbe MW (2005) The concept of quality of life in dementia in the different stages of the disease. Int Psychogeriatr 17, 353-370.

[11] Banerjee S, Samsi K, Petrie CD, Alvir J, Treglia M, Schwam EM, del Valle M (2009) What do we know about quality of life in dementia? A review of the emerging evidence on the predictive and explanatory value of disease specific measures of health related quality of life in people with dementia. Int J Geriatr Psychiatry 24, 15-24.

[12] Martyr A, Nelis SM, Quinn C, Wu Y-T, Lamont RA, Henderson C, Clarke R, Hindle JV, Thom JM, Jones IR (2018) Living well with dementia: A systematic review and correlational meta-analysis of factors associated with quality of life, well-being and life satisfaction in people with dementia. Psychol Med 48, 2130-2139.

[13] Gobbens R, Van Assen M (2014) The prediction of quality of life by physical, psychological and social components of frailty in community-dwelling older people. Qual Life Res 23, 2289-2300. 
[14] Selwood A, Thorgrimsen L, Orrell M (2005) Quality of life in dementia-a one-year follow-up study. Int J Geriatr Psychiatry 20, 232-237.

[15] Mjørud M, Røsvik J, Rokstad AMM, Kirkevold M, Engedal $\mathrm{K}$ (2014) Variables associated with change in quality of life among persons with dementia in nursing homes: A 10 months follow-up study. PLoS One 9, e115248.

[16] Livingston G, Cooper C, Woods J, Milne A, Katona C (2008) Successful ageing in adversity-The LASER-AD longitudinal study. J Neurol Neurosurg Psychiatry 79, 641-645.

[17] Haaksma ML, Leoutsakos J-MS, Bremer JA, Aalten P, Ramakers IH, Verhey FR, Rikkert MGO, Melis RJ (2018) The clinical course and interrelations of dementia related symptoms. Int Psychogeriatr 30, 859-866.

[18] Tatsumi H, Nakaaki S, Torii K, Shinagawa Y, Watanabe N, Murata Y, Sato J, Mimura M, Furukawa TA (2009) Neuropsychiatric symptoms predict change in quality of life of Alzheimer disease patients: A two-year follow-up study. Psychiatry Clin Neurosci 63, 374-384.

[19] Boström F, Jönsson L, Minthon L, Londos E (2007) Patients with dementia with Lewy bodies have more impaired quality of life than patients with Alzheimer disease. Alzheimer Dis Assoc Disord 21, 150-154.

[20] Aalten P, Ramakers IH, Biessels GJ, De Deyn PP, Koek HL, OldeRikkert MG, Oleksik AM, Richard E, Smits LL, van Swieten JC (2014) The Dutch Parelsnoer InstituteNeurodegenerative diseases; methods, design and baseline results. BMC Neurol 14, 254.

[21] Manniën J, Ledderhof T, Verspaget HW, Snijder RR, Flikkenschild EF, van Scherrenburg NP, Stolk RP, Zielhuis GA (2017) The Parelsnoer institute: A national network of standardized clinical biobanks in the Netherlands. Open J Bioresour 4, 3.

[22] van der Flier WM, Scheltens P (2018) Amsterdam Dementia Cohort: Performing research to optimize care. J Alzheimers Dis 62, 1091-1111.

[23] McKeith I, Dickson DW, Lowe J, Emre M, O’brien J, Feldman H, Cummings J, Duda J, Lippa C, Perry E (2005) Diagnosis and management of dementia with Lewy bodies third report of the DLB consortium. Neurology 65, 18631872.

[24] McKhann G, Drachman D, Folstein M, Katzman R, Price D, Stadlan EM (1984) Clinical diagnosis of Alzheimer's disease Report of the NINCDS-ADRDA Work Group* under the auspices of Department of Health and Human Services Task Force on Alzheimer's Disease. Neurology 34, 939-939.

[25] Lamers LM, Stalmeier PFM, McDonnell J, Krabbe PFM, van Busschbach JJ (2005) Kwaliteit van leven meten in economische evaluaties: Het Nederlands EQ-5D-tarief. Ned Tijdschr Geneeskd 149, 1574-1578.

[26] Cummings JL (1997) The Neuropsychiatric Inventory Assessing psychopathology in dementia patients. Neurology 48, 10S-16S.

[27] Fish J (2011) Disability assessment for dementia. In Encyclopedia of Clinical Neuropsychology. Springer, pp. 860-862.
[28] Sheikh JI, Yesavage JA (1986) Geriatric Depression Scale (GDS): Recent evidence and development of a shorter version. Clin Gerontol 5, 165-173.

[29] Folstein MF, Folstein SE, McHugh PR (1975) "Mini-mental state": A practical method for grading the cognitive state of patients for the clinician. J Psychiatr Res 12, 189-198.

[30] Heggie M, Morgan D, Crossley M, Kirk A, Wong P, Karunanayake C, Beever R (2012) Quality of life in early dementia: Comparison of rural patient and caregiver ratings at baseline and one year. Dementia 11, 521-541.

[31] Selwood A, Thorgrimsen L, Orrell M (2005) Quality of life in dementia-a one-year follow-up study. Int J Geriatr Psychiatry 20, 232-237.

[32] Savva GM, Zaccai J, Matthews FE, Davidson JE, McKeith I (2009) Prevalence, correlates and course of behavioural and psychological symptoms of dementia in the population. $\mathrm{Br}$ J Psychiatry 194, 212-219.

[33] Kim H, Chang M, Rose K, Kim S (2012) Predictors of caregiver burden in caregivers of individuals with dementia. $J$ Adv Nurs 68, 846-855.

[34] Lee DR, McKeith I, Mosimann U, Ghosh-Nodyal A, Thomas AJ (2013) Examining carer stress in dementia: The role of subtype diagnosis and neuropsychiatric symptoms. Int J Geriatr Psychiatry 28, 135-141.

[35] Rongve A, Vossius C, Nore S, Testad I, Aarsland D (2014) Time until nursing home admission in people with mild dementia: Comparison of dementia with Lewy bodies and Alzheimer's dementia. Int J Geriatr Psychiatry 29, 392-398.

[36] Gaugler JE, Yu F, Krichbaum K, Wyman JF (2009) Predictors of nursing home admission for persons with dementia. Med Care 47, 191-198.

[37] Bharucha AJ, Pandav R, Shen C, Dodge HH, Ganguli M (2004) Predictors of nursing facility admission: A 12-year epidemiological study in the United States. J Am Geriatr Soc 52, 434-439.

[38] Harris Y, Cooper JK (2006) Depressive symptoms in older people predict nursing home admission. J Am Geriatr Soc 54, 593-597.

[39] Winter Y, Korchounov A, Zhukova TV, Bertschi NE (2011) Depression in elderly patients with Alzheimer dementia or vascular dementia and its influence on their quality of life. J Neurosci Rural Pract 2, 27.

[40] Andersen CK, Wittrup-Jensen KU, Lolk A, Andersen K, Kragh-Sørensen P (2004) Ability to perform activities of daily living is the main factor affecting quality of life in patients with dementia. Health Qual Life Outcomes 2, 52.

[41] Slot RE, Verfaillie SC, Overbeek JM, Timmers T, Wesselman LM, Teunissen CE, Dols A, Bouwman FH, Prins ND, Barkhof F (2018) Subjective Cognitive Impairment Cohort (SCIENCe): Study design and first results. Alzheimers Res Ther 10, 76 .

[42] Larsson V, Engedal K, Aarsland D, Wattmo C, Minthon L, Londos E (2011) Quality of life and the effect of memantine in dementia with lewy bodies and Parkinson's disease dementia. Dement Geriatr Cogn Disord 32, 227-234. 